\title{
IEEE 802.11 based Medium Access Design for Wireless loT-Blockchain Networks
}

Graduate Students,

Arezou Zamanyzadeh Abyaneh, Nizar Zorba

Information and Communication Technologies (ICT)

Department of Electrical Engineering, Qatar University, Doha, Qatar

\section{Abstract}

Communication is a very basic essence of the blockchain network and must be carefully planned while integrating with loT, where an extremely large number of devices are interconnected. In this work, blockchain nodes are assumed to use wireless channels to communicate among themselves and other elements of the loT setup. These communications can be in unicast and broadcast manner where transmission latency and throughput are significant metrics that might jeopardize the overall system. This work is proposing a Medium Access Control (MAC) mechanism addressing these performance metrics and best suitable for wireless loT-Blockchain system. The proposed MAC protocol is based on the widely used IEEE 802.11 protocol, Carrier Sense Multiple Access/Collision Avoidance (CSMA/CA) basic access.

\section{MAC for Wireless loT-Blockchain}

The setup in proposed MAC is controlled by the leading node that has a collection of transactions that it wishes to add to blockchain, and in this case, it will send the transaction through a wireless channel as a broadcast packet. Therefore, after a short interframe space (SIFS), and upon of successful transmission, the full nodes will start the validation process using the consensus protocol, and each node sends back acknowledgment (ACK) frames confirming the validity and safety of the transaction immediately after a SIFS. After successfully receiving all the ACK frames, the leading full node will wait for a period equal to distribute interframe space (DIFS), for guarantee the channel to be idle in order to start a new transmission [2]

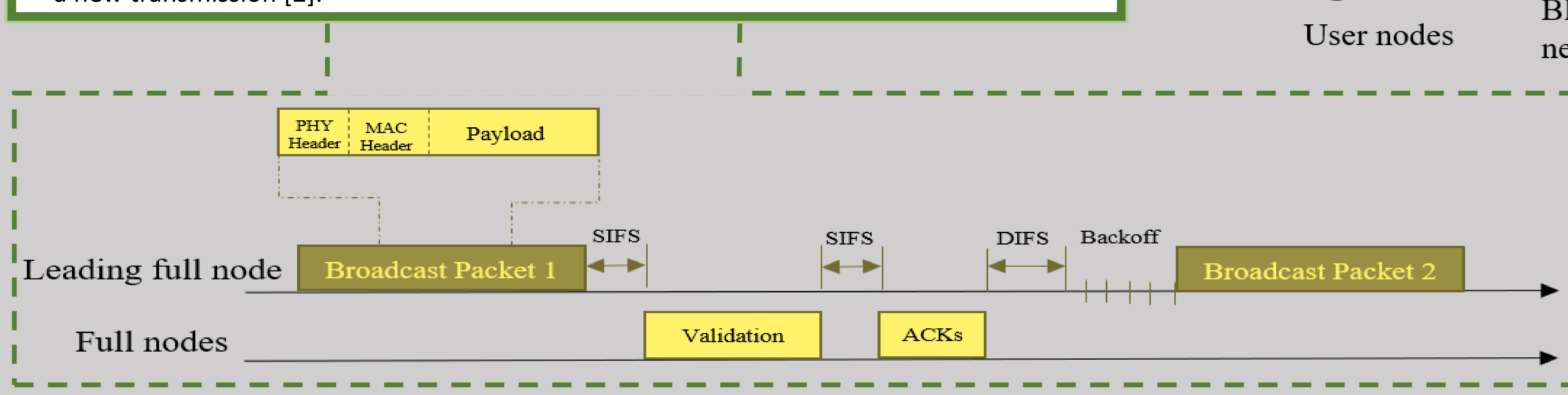

\section{Wireless loT-Blockchain SYSTEM MODEL}

The main process to convert a raw data originated from user node (IoT device) into a confirmed block through a full node (blockchain node) is denoted as: (i) A user node acquires new unsigned transactions

(ii) User node send the transaction to multiple full nodes.

(iii) A full node claims the transactions following the Ethereum protocol

(iv) The leading full node broadcasts the transaction using CSMA/CA to all blockchain nodes to confirm its legitimacy.

(v) If most full nodes validate the transaction following PoS consensus, the transaction will be added to a block, if not it will be declined.

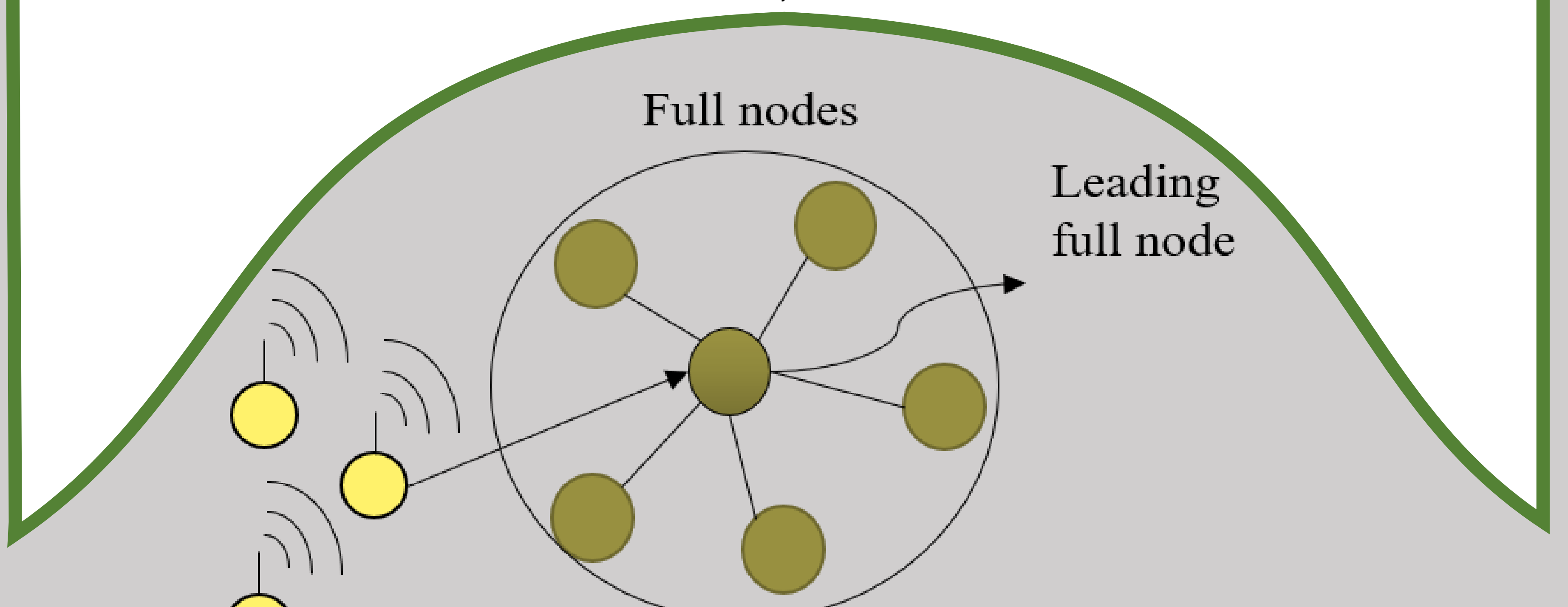

Blockchain network

\section{Mathematical Modeling}

Total delay, $T_{s}^{t}=E[P]+P H Y_{\text {header }}+M A C_{\text {header }}+S I F S+N \times \frac{E[P]}{E[T]} T_{d}+S I F s+N \times A C K s+D I F S+\delta$

$$
\text { Collision delay, } T_{c}=E[P]+P H Y_{\text {header }}+M A C_{\text {header }}+D I F S+\delta
$$

$$
\text { Throughput }=\frac{P_{S} E[P]}{P_{S} T_{S}+\left(1-P_{c}\right) T_{c}}
$$

Note: $P_{S}$ is the probability that a packet is successfully transmitted and $P_{c}$ the probability that a transmitted packet collides which is defined in detail in [1]

\section{Evaluation and Simulation Results}

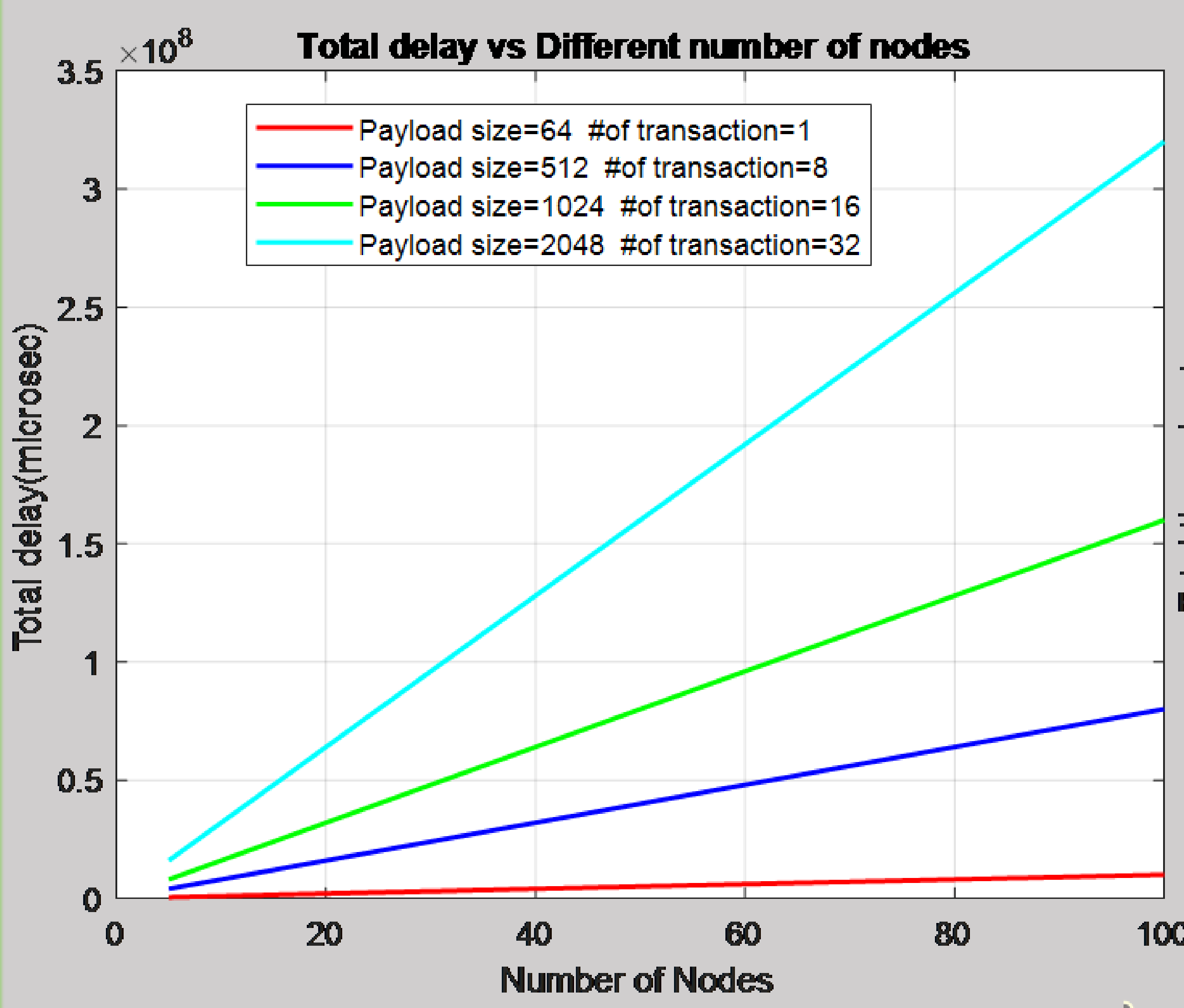

Delay Vs \#of Nodes

cor more Hof Nodes

Reasons: Validation Delay

wever, more validating nodes means more security!

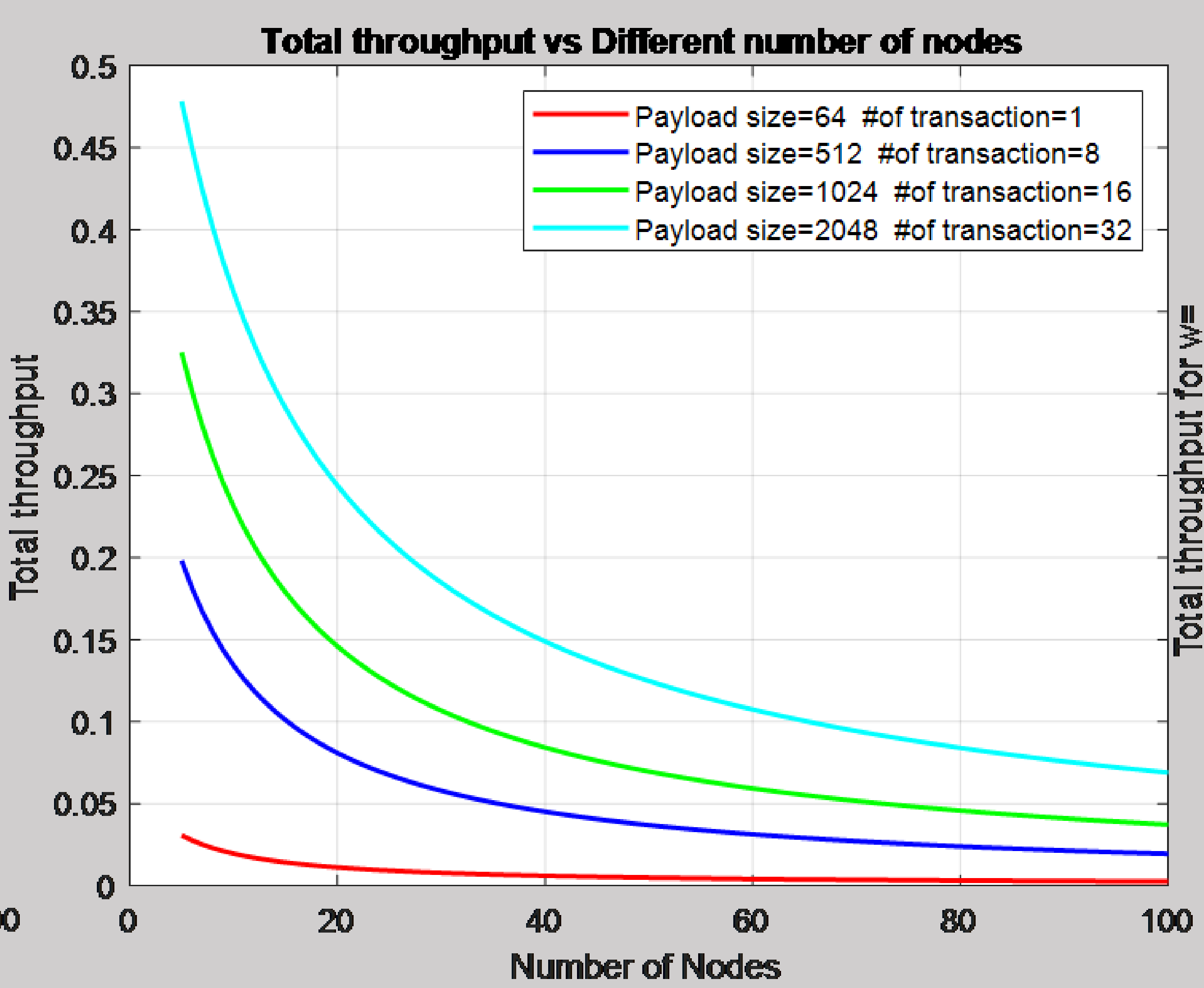

Throughput Vs \#of Nodes with constant CW

Throughput decreases for more \#of Nodes

Reasons: increase \#of consecutive ideal slots between two broadcasts

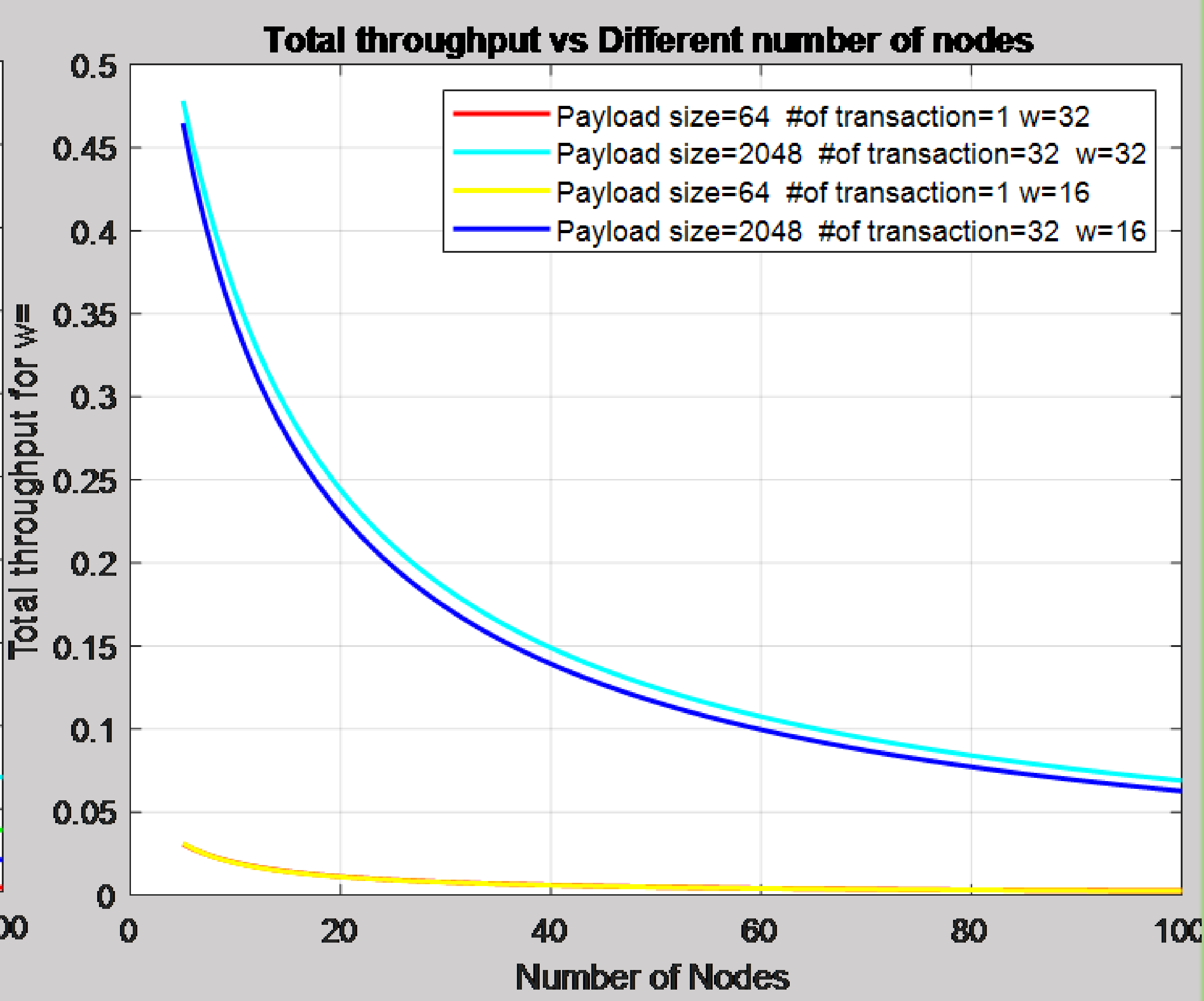

Throughput Vs \#of Nodes for Variable CW Throughput decreases as Decreasing the window size. Reasons: high probability of collision effect will be more apparent with increasing the payload

\section{Conclusion}

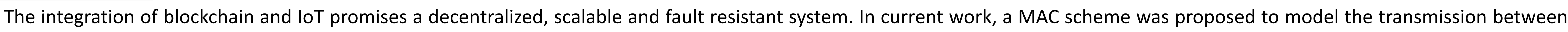

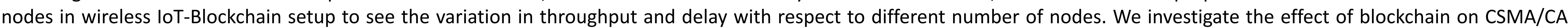

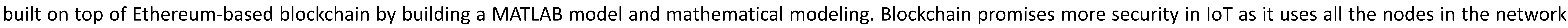

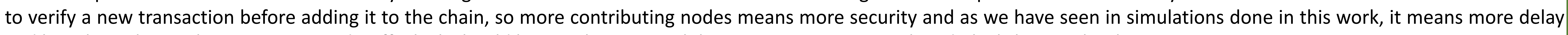
and less throughput. This presents a trade-off which should be a real concern while integrating loT network and Blockchain technology. References

[1] G. Bianchi, "Performance analysis of the IEEE 802.11 distributed coordination function," IEEE J. Sel. Areas Commun., vol. 18, no. 3, pp. 535-547, 2000. 